\title{
The contribution of flux transfer events to convection
}

\author{
Article \\ Published Version
}

Lockwood, M., Cowley, S. W. H., Smith, M. F., Rijnbeek, R. P. and Elphic, R. C. (1994) The contribution of flux transfer events to convection. Geophysical Research Letters, 22 (10). pp. 1185-1188. ISSN 00948276 doi:

https://doi.org/10.1029/95GL01008 Available at https://centaur.reading.ac.uk/38805/

It is advisable to refer to the publisher's version if you intend to cite from the work. See Guidance on citing.

Published version at: http://dx.doi.org/10.1029/95GL01008

To link to this article DOI: http://dx.doi.org/10.1029/95GL01008

All outputs in CentAUR are protected by Intellectual Property Rights law, including copyright law. Copyright and IPR is retained by the creators or other copyright holders. Terms and conditions for use of this material are defined in the End User Agreement.

\section{www.reading.ac.uk/centaur}

\section{CentAUR}

Central Archive at the University of Reading 
Reading's research outputs online 


\title{
The contribution of flux transfer events to convection
}

\author{
M. Lockwood ${ }^{1}$, S,W.H. Cowley ${ }^{2}$, M.F. Smith ${ }^{3}$, R.P. Rijnbeek ${ }^{4}$ and R.C. Elphic ${ }^{5}$
}

Abstract. It is shown from flux transfer event (FTE) occurrence statistics, observed as a function of MLT by the ISEE satellites, that recent 2-dimensional analytic theories of the effects of pulsed Petschek reconnection predict FTEs to contribute between 50 and $200 \mathrm{kV}$ to the total reconnection voltage when the magnetosheath field points southward. The upper limit $(200 \mathrm{kV})$ allows the possibility that FTEs provide all the antisunward transport of open field lines into the tail lobe. This range is compared with the voltages associated with series of FTEs signatures, as inferred from ground-based observations, which are in the range $10-60 \mathrm{kV}$. We conclude that the contribution could sometimes be made by a series of single, large events; however, the voltage is often likely to be contributed by several FTEs at different MLT.

\section{Introduction}

To date, no observations at the dayside magnetopause have been made which define the open magnetic flux transported from the dayside to the tail lobe in individual flux transfer events (FTEs). However, we can use FTE occurrence statistics to estimate the collective contribution of all FTEs to magnetospheric and ionospheric convection. This is done here in section 4 , after a discussion of the reconnection theory of magnetopause FTE signatures and of what information can (and cannot) be gained from them (section 2). Limits to the dimensions and voltage contribution of FTEs have recently been proposed by Newell and Sibeck [1993] and these are discussed in section 3 . In section 5 we briefly discuss the implications of ground-based observations of putative FTE signatures in the ionosphere.

\section{What Can We Infer from Magnetopause FTE Observations?}

Figure 1 shows schematically a cross-section the reconnection layer (i.e. the open LLBL) of newly-opened field lines at the dayside magnetopause. The $\mathrm{N}$ axis is the outward boundary-normal direction and the $\mathrm{L}^{\prime}$ axis shown lies in the magnetopause and is the direction of motion of newly-opened field lines as they evolve under magnetic

\footnotetext{
${ }^{1}$ Rutherford Appleton Laboratory, Chilton, Oxfordshire, U.K.

${ }^{2}$ Blackett Laboratory, Imperial College, London, U.K.

3 Laboratory for Extraterrestrial Physics, Goddard SFC, Greenbelt, Maryland, U.S.A.

${ }^{4}$ Space Science Centre, Sussex University, Falmer, U.K.

${ }^{5}$ Los Alamos National Laboratory, New Mexico, U.S.A.
}

Copyright 1995 by the American Geophysical Union.

Paper number 95GL01008

0094-8534/95/95GL-01008\$03.00 tension and magnetosheath flow. A bipolar signature in the boundary-normal field $B_{N}$ is seen in an FTE as a thickening in the magnetopause reconnection layer propagates across the satellite, S. This conceptual picture of FTEs was suggested by Biernat et al. [1987] and Southwood et al. [1988] and has been verified in 2-D MHD simulations [Scholer, 1988] and by analytic theory [Semenov et al. 1992]. Adding an $\mathbf{M}^{\prime}$ component of the newly-opened flux produces a topology more like the original 3-D "elbow" model of Russell and Elphic [1978] (see review by Sonnerup, 1988]).

The velocity of newly-opened field lines along the magnetopause (the de Hoffman-Teller velocity, $V$ ) is, to a good approximation, independent of the reconnection rate. Hence the motion of the FTE is the same as that of all the other newly-opened field lines produced by the same segment of the $\mathrm{X}$-line. From the duration of an event $\delta t$ and the velocity $V$, we can estimate its dimension in the direction of motion (L') to be $a=V . \delta t$ (typically $200 \mathrm{~km} \mathrm{~s}^{-1} \times 100 \mathrm{~s} \approx 3 R_{E}$ [Russell and Elphic, 1978; Rijnbeek et al., 1984]). Furthermore, from comparisons of data from the two ISEE spacecraft at different distances from the magnetopause, Saunders et al. [1984] derived the boundary-normal (N) extent of these events $(c)$ to be also of order $1 R_{E}$. However, the extent $b^{\prime}$ of the third dimension of the FTE signature (in the $\mathrm{M}^{\prime}$ direction, transverse to the FTE motion) is completely unknown and could only be determined using many spacecraft which were simultaneously at the magnetopause - a measurement which has not yet been possible. We need to differentiate between $b^{\prime}$ and $b$, the latter being the extent in the $\mathrm{M}^{\prime}$ direction of the patch of enhanced magnetic field threading the magnetopause. The elbow model predicts $b$ is only slightly smaller than $b^{\prime}$ and 2-D models give $b=b^{\prime}$. Russell and Elphic [1978] effectively employed an estimate for $b$ by adopting a paradigm in which the FTE flux tube is roughly circular. The 2-D models and theory, however, make it clear that there is no need to make this assumption. This is because they predict that the FTE structure observed depends only on the reconnection rate variation at the element of the $\mathrm{X}$-line which is on the same streamline as the satellite: the satellite gains no information on what happens at other segments of the Xline because the streamlines take the resulting open flux tubes elsewhere. This means that not only do we not know $b$, but we do not know if there are other FTEs travelling on

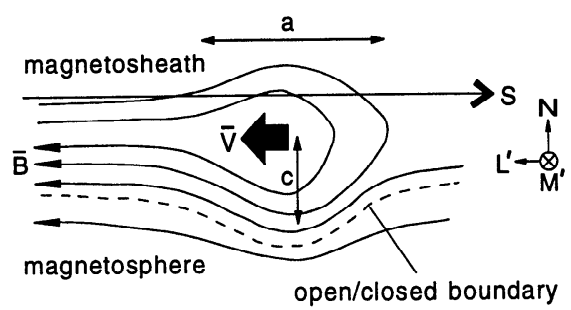

Fig. 1. Cross section of an FTE for a 2-dimensional model, with newly-opened field lines $\underline{B}$ moving at velocity $\underline{V}$ over the magnetopause. $\mathrm{S}$ is a locus of a satellite in the FTE rest frame. 


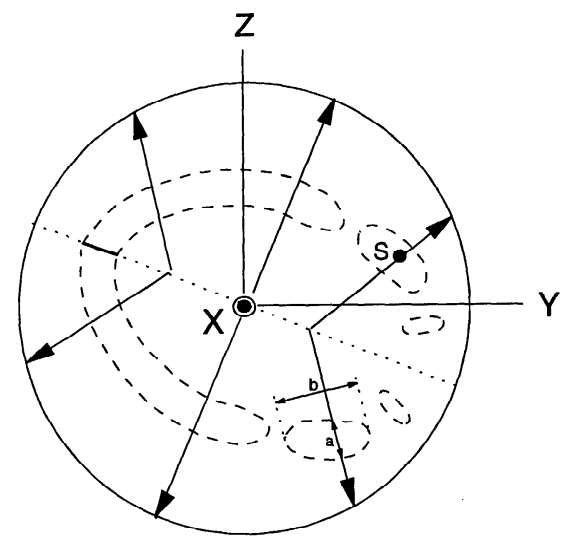

Fig. 2. Schematic of the dayside magnetopause seen from the sun. The dashed lines bound regions of enhanced flux threading the boundary (associated with FTEs), the arrows are flow streamlines over the boundary and the dotted line is where reconnection takes place.

other streamlines. The repetitive cycle of these other FTEs need have no relation to the cycle observed by the satellite.

Figure 2 illustrates some of the possibilities which may occur. This schematic views the dayside magnetopause from the sun and shows patches, bounded by the dashed lines, in which enhanced flux threads the boundary (produced by bursts of enhanced reconnection and associated with the FTE signature as shown by figure 1). All reconnection is taken to occur along the dotted line and the solid arrows show the streamlines on the boundary along which the newly-opened field lines evolve under magnetic tension and the magnetosheath flow [see Cowley and Owen, 1989]. (Note that figure 1 shows a cross-section of the magnetopause along one of these streamlines). These flow streamlines are approximately independent of the reconnection rate. Figure 2 shows three patches of enhanced boundary-normal field, the middle one of which passes over a satellite at $S$ in the northern hemisphere, and has dimensions $a$ and $b$ along and normal to the flow (as shown for the corresponding patch in the southern hemisphere). As discussed above, the satellite $\mathrm{S}$ cannot determine the dimension $b$. Neither can $\mathbf{S}$ know about the other 'side-by-side' FTEs on other streamlines: in figure 2, two such events are shown, one of very small $b$ to the dusk side of $\mathrm{S}$ and one of extremely large $b$ to the dawn side of S. Note that latter patch is here still growing in $b$ as a pulse of enhanced reconnection propagates along the $\mathrm{X}$-line towards dawn. This possibility has been suggested from ionospheric observations, as discussed by Lockwood [1994].

Statistically, we do know the extent of the region on the magnetopause where FTEs are observed. Figure 3 plots the occurrence probability, $f$, of magnetopause crossings during which one or more magnetosheath FTEs were observed. These data are from the survey of ISEE data by Rijnbeek et al. [1984], but only include passes when the observed magnetosheath field was classified as southward. This plot differs from those given in the original paper only in that it shows this occurrence frequency as a function of MLT. Also shown is the number of crossings with the required southward sheath field in each 2-hour MLT sector. It can be seen that the occurrence of at least one FTE was high when the sheath field was southward ( $f$ in the range 0.7-0.9) throughout the dayside magnetopause (5-19 MLT).

\section{Suggested limits to the voltage contribution of FTEs}

The open flux transported into the tail lobe by an individual FTE is $\Delta F=\int_{0}^{a} \int_{0}^{b} B_{N} d a d b$ and the voltage contribution to convection is $\langle\Delta F / \Delta t\rangle$, where $\Delta t$ is the repeat period of events [Russell and Elphic, 1978; Lockwood et al., 1990; Elphic et al., 1990; Lockwood and Cowley, 1994]. Newell and Sibeck [1993] have attempted to devise two limits to the unknown FTE dimension $b$ and hence to the events' contribution to convection. In this section we show that both these limits are unfounded and inconsistent with FTE occurrence statistics, as shown in figure 3, for recent theories of FTEs.

The first limit placed by Newell and Sibeck quotes a rough estimate that all FTEs are reconnected within $6 R_{E}$ of the subsolar point. We here note that flow streamlines would have to diverge from such reconnection sites so that the FTEs are seen over the whole dayside magnetopause at higher magnetic latitudes: even the ISEE survey did not define the full local time extent of FTE occurrence and they were found from Y of -15 $R_{E}$ to $+15 R_{E}$ [figure 5, Rijnbeek et al., 1984]. For a typical dayside magnetopause, this gives a minimum extent of FTE observation of order $45 R_{E}$. Hence $b$ could, in principle, be this large for each event. The total flux in the event $\Delta F$ does not alter once the event is fully detached from the $\mathrm{X}$-line and hence, if events do subsequently elongate (i.e. $b$ increases) under diverging flow, then the $B_{N}$ and $a$ must have decreased between the subsolar reconnection site and the observation sites at mid-latitudes. Newell and Sibeck combine the FTE characteristics (the interior field and dimension $a$ ) measured at middle latitudes with this estimated maximum extent of the $\mathrm{X}$-line (i.e. maximum $b$ ) in the equatorial region. This is inconsistent: one should employ the maximum $b$ at middle latitudes, from where are derived the other estimates. In other words, one should use at least $45 R_{E}$ instead of $12 R_{E}$ and hence the limit of Newell and Sibeck should be at least 3.75 times larger (i.e. $>142 \mathrm{kV}$ ).

A second, even smaller, limit on $\Delta F / \Delta t$ was placed by Newell and Sibeck by stating the whole FTE must be reconnected in a 2 min. period. A corresponding coherence argument about the voltage along O-lines was given by

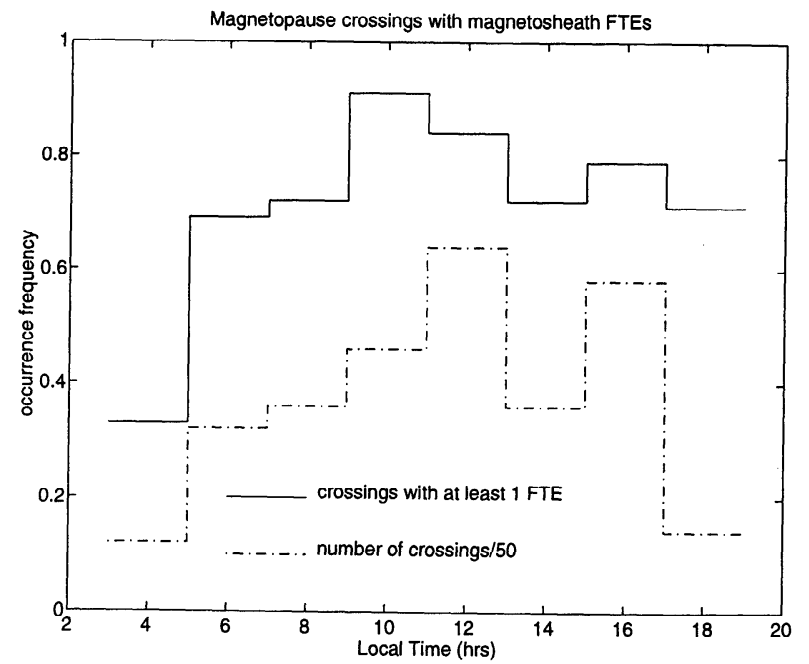

Fig. 3. The fraction $f$ (of $N$ magnetopause crossings by the ISEE satellites for southward magnetosheath field) with at least one FTE. The solid and dot-dash lines give $f$ and $N / 50$, respectively, for 2-hour MLT bins. 
Vasyliunas [1980]. The value of $2 \mathrm{~min}$. derives from the observed FTE durations, $\delta t$, and it shows that the reconnection pulse lasted $\delta t$, at the point on the $\mathrm{X}$ line which reconnected the observed part of the FTE. Elsewhere on the $\mathrm{X}$ line the reconnection pulse may last for an interval $8 t$ also, but this does not have to be the same interval, as Newell and Sibeck assume. Neither is there a requirement that the different events in figure 2 be reconnected in the same 2 min. interval. Newell and Sibeck argue that unless the whole FTE is reconnected within the interval $8 t$, it will not behave as a coherent whole. This is neither true nor relevant. It is not true because the flow characteristics from any one point on an X-line will depend upon the field curvature and the sheath flow, both of which will vary along its length in a continuous manner. This being the case, the fossil FTE signature will evolve away from the reconnection site in a way which varies continuously along its length, no matter what that length may be. However, whether or not events move as coherent entities along different streamlines after reconnection is of no relevance to the magnetopause observations, which can only sample an event along the one streamline. In addition, this makes no difference to the total flux reconnected and to the voltage contribution. If one adopts a smaller $b^{\prime}$, one also has to invoke more side-by-side events (in inverse proportion to $b^{\prime}$ ) to explain the event occurrence statistics. Newell and Sibeck's limit of $14.5 \mathrm{kV}$ to the voltage contribution of FTEs, $\Delta F / \Delta t$, assumes that there exists on the magnetopause only one event of $b=4.6 \mathrm{R}_{\mathrm{E}}$ every $8 \mathrm{~min}$. From the 2-D FTE theory, an event of length $b^{\prime}=b=4.6 \mathrm{R}_{\mathrm{E}}$ would only be seen over a small fraction of the dayside magnetopause. This is in direct contradiction of FTE occurrence statistics. FTEs were observed with the mean period of about $8 \mathrm{~min}$. (for passes with southward sheath field) at all points on the magnetopause surveyed by Rijnbeek et al. [1984], from the dawn to the dusk flank (i.e. over a spatial extent of at least $45 \mathrm{R}_{\mathrm{E}}$ ). If there was just one event of $b=$ $4.6 \mathrm{R}_{\mathrm{E}}$ every $8 \mathrm{~min}$., this would require that the signature always passed over the satellite, wherever it happened to be on the dayside magnetopause. The only explanations for such behaviour would be either that the spacecraft was triggering the FTEs or that $b^{\prime}$ is an order of magnitude larger than $b$. We conclude that either events are bigger than $b^{\prime}=4.6 \mathrm{R}_{\mathrm{E}}$ wide or there are several of them on the magnetopause at the same time. Newell and Sibeck state that the second possibility would mean the repeat period of observed FTEs would be reduced but this is not the case because 'side-by-side' FTEs are on completely different flow streamlines, only one of which passes over the spacecraft. Either way, the contribution to the transpolar voltage of FTEs is greater than the upper limit of Newell and Sibeck. In the next section this argument is inverted so that the FTE occurrence statistics are used to evaluate the voltage contribution of FTEs.

\section{The contribution of FTEs to convection}

We here use $\Phi$ to denote the flux threading the magnetopause which crosses unit length in the $\mathbf{M}^{\prime}$ direction, associated with the passage of an FTE signature. Hence $\Delta F$ is equal to $b \boldsymbol{\Phi}$. As discussed above, the dimension $b$ is not known but $\boldsymbol{\Phi}$ can be estimated from the satellite observations of FTEs. Considering figure 1 , we find that the flux threading the magnetopause, per unit length in the $\mathbf{M}^{\prime}$ direction, is the interior field, $B_{f t e}$, integrated over the dimension $c$. If $B_{f t e}$ is constant this reduces to $c B_{f t e}$. We here take a typical value of $c$ of $1 R_{E}$ along with a conservative estimate for $B_{f t e}$ of $20 \mathrm{nT}$, giving $\Phi$ of $0.13 \mathrm{~Wb} \mathrm{~m}^{-1}$. This is smaller than the $0.24 \mathrm{~Wb}$ $\mathrm{m}^{-1}$ used by Newell and Sibeck because they used a larger $B_{f t e}$. It must be remembered that these estimates of mean $\Phi$ are crude and we would regard our value as accurate to within a factor of roughly two. This is consistent with Newell and Sibeck's $\Phi$ in that they were attempting to derive an upper limit. The mean open flux being carried over a satellite, S, by FTEs per unit length per unit time during southward IMF is $f \Phi b /\left(b^{\prime} \Delta t\right)$ where, as above, $f$ is the probability that there are FTE signatures on any one crossing and $\Delta t$ is their mean repetition period. By Faraday's law, this is the FTE voltage contribution per unit $\mathbf{M}^{\prime}$ length. Hence the voltage contribution of FTEs in an MLT sector is

$$
\Delta V=f \Phi d b /\left(b^{\prime} \Delta t\right)
$$

where $d$ is the length of the magnetopause covered by that MLT sector, in the direction normal to the FTE motion. We here use the 2-D FTE models, for which $b / b^{\prime}$ has a maximum value of 1 , giving an upper limit to the voltage (which can be compared with the upper limit derived by Newell and Sibeck). The total voltage is the sum of the $\Delta V$ for all the MLT sectors. Figure 3 gives the variation of $f$ with MLT and figure 4 shows the corresponding average values of $d$ and $\Delta t$. The mean $\Delta t$ are taken from a survey of intervals between magnetosheath FTEs, as observed in the same ISEE dataset when the sheath field was southward by Lockwood and Wild [1993]. The value of $d$ for each MLT sector was derived by fitting a model magnetopause to all the magnetopause crossings in that sector during southward sheath field. The value of $d$ used was the length of the line in this model magnetopause, subtended by the MLT sector at the mean latitude of the crossings. Figure (4) shows $\Delta V$ is relatively independent of MLT and that, despite the lower $f$ near dawn and dusk, $\Delta V$ is large there because of the smaller $\Delta t$ and greater $d$.

The last step is to integrate over the entire dayside magnetopause by summing the contributions from FTEs in all the local time sectors. From figure 4 this total contribu-

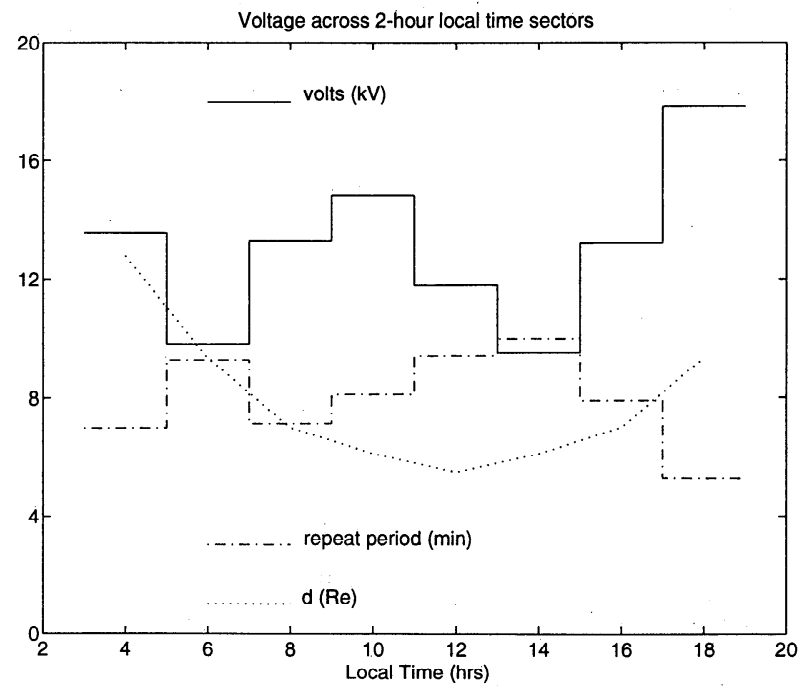

Fig. 4. The mean repeat period of FTEs $\Delta t$ (in min. - dot-dash line), distance $d$ (in $\mathrm{R}_{E}$ - dotted line) and the voltage $\Delta V$ (in $\mathrm{kV}$ - solid line) for 2-hour MLT bins. 
tion of FTEs to convection (for southward magnetosheath field and over the 3-19 MLT region covered by the survey) is $104 \mathrm{kV}$. Adoption of Newell and Sibeck's value for $\Phi$ would raise this estimate to $192 \mathrm{kV}$. The estimated uncertainty in $\Phi$ gives the total voltage contribution of FTEs to be in the range $52-208 \mathrm{kV}$. From this we can make two statements. Firstly, estimates which restrict this contribution to below about $50 \mathrm{kV}$ are inconsistent with FTE occurrence statistics and recent FTE theories. Secondly, because the average reconnection voltage during southward IMF is typically less than about $130 \mathrm{kV}$, it is certainly not impossible that FTEs provide all the reconnection voltage, and hence nearly all of the convection transpolar voltage.

This estimated range of voltages has been based on the occurrence frequency of FTEs and does not rely on an estimate of the unknown dimension of each FTE, $b$. The occurrence probability, $f$, repeat period, $\Delta t$, and hence the total voltage contribution, could have originated from a series of FTEs of huge $b$ which cover almost the entire dayside magnetopause, or from very many FTEs of very small $b$ which individually cover only a tiny fraction of the magnetopause. These two extreme possibilities can give the same total contribution of FTEs to convection, they differ only in the contribution of individual events to the total. Hence the following debate about the unknown dimension of individual FTEs does not alter the above conclusions.

\section{Ground-based observations}

The importance of ionospheric observations is that, once FTE signatures can be recognised, both the dimensions $a$ and $b$ can be determined. In addition, the ionospheric field is effectively constant and known, and hence the flux transferred by individual FTEs (as opposed to the average contribution of all FTEs determined in the previous section) can be estimated. This argument has been used to estimate voltages in the range 10-60 kV [Elphic et al., 1990; Lockwood et al., 1990; 1993a; Pinnock et al., 1993; A.S. Rodger, private communication, 1994]. Newell and Sibeck [1994] argue that the larger of these estimates are in error due to slant path effects on $630 \mathrm{~nm}$ optical observations; however such effects can act to make the apparent size of the events too small as well as too big, depending on the luninosity contour chosen to delineate the event. The contours employed by Lockwood et al. [1993a] were chosen so as to make the latitudinal width and speed of the optical event match those from the EISCAT radar data, which are not subject to this effect. In all these cases there may have been other FTEs at MLTs not covered by the ground-based observations, or a background reconnection rate between the pulses. It has been shown in the previous section that the total contribution of FTEs can be in the range $50-200 \mathrm{kV}$, and hence it is possible that sometimes this is produced by just one single large event per repetition period. However, if the total contribution exceeds about $60 \mathrm{kV}$, this may imply a number of side-by-side events at different MLT.

\section{References}

Biernat, H. K., M. F. Heyn, and V. S. Semenov, Unsteady Petschek reconnection, J. Geophys. Res., 92, 3392, 1987.

Cowley, S.W.H. and C.J. Owen, A simple illustrative model of open flux tube motion over the dayside magnetopause, Planet. Space Sci., 37, 1461, 1989.

Elphic, R.C., et al., Flux transfer events at the magnetopause and in the ionosphere, Geophys. Res. Lett., 17, 2241-2244, 1990.

Lockwood, M., Ionospheric signatures of pulsed magnetopause reconnection, in "Physical signatures of magnetopause boundary layer Processes", ed. J.A. Holtet and A. Egeland, NATO ASI Series C, Vol. 425, Kluwar, 229-243, 1994.

Lockwood, M. and S.W.H. Cowley, Comment on "Ionospheric signatures of dayside magnetopause transients: a case study using satellite and ground measurements" by Denig et al., J. Geophys. Res., 99, 4253, 1994.

Lockwood, M., and M.N. Wild, On the quasi-periodic nature of magnetopause flux transfer events, J. Geophys. Res., 98, 5935, 1993.

Lockwood, M., et al., The ionospheric signatures of flux transfer events and solar wind dynamic pressure changes, J. Geophys. Res., 95, 17,113, 1990.

Lockwood, M., et al., The implications of the altitude of transient $630 \mathrm{~nm}$ dayside auroral emissions, J. Geophys. Res., 98, 15571, 1993.

Newell, P.T. and D.G. Sibeck, Upper limits on the contribution of flux transfer events to ionospheric convection, Geophys. Res. Lett., 20, 2829, 1993.

Pinnock, M., et al., Observations of an enhanced convection channel in the cusp ionosphere, J.Geophys.Res.,98, 3767,1993

Rijnbeek, R. P., et al., A survey of dayside flux transfer events observed by the ISEE 1 and 2 magnetometers, J. Geophys. Res., 89, 786, 1984.

Russell, C.T., and R.C. Elphic, Initial ISEE magnetometer results Magnetopause observations, Space Sci. Rev., 22, 681, 1978.

Saunders, M. A., et al., Flux transfer events, scale size and interior structure, Geophys. Res. Lett., 11, 131, 1984.

Scholer, M., Magnetic flux transfer at the magnetopause based on single X-line bursty reconnection, Geophys. Res. Lett., 15, 291, 1988.

Semenov, V.S., et al., Time-dependent localized reconnection of skewed magnetic fields, J. Geophys. Res., 97, 4251, 1992.

Sonnerup, B.U.O., Experimental tests of FTE theories, $A d v$. Space Res., 8(9), 263-272, 1988.

Southwood, D.J., C.J. Farrugia, and M.A. Saunders, What are flux transfer events?, Planet. Space Sci., 36, 503, 1988.

Vasyliunas, V.M., Upper limit on the electric field along a magnetic O line, J. Geophys. Res., 85, 4616, 1980.

S.W.H. Cowley, Blackett Laboratory, Imperial College, London, SW7 2BZ, UK.

R.C. Elphic, Los Alamos National Laboratory, Los Alamos, New Mexico, 87545, USA.

M. Lockwood, Rutherford Appleton Laboratory, Chilton, Didcot, OX11 0QX, UK. (c-mail: mike@eiscat.ag.rl.ac.uk)

R.P. Rijnbeek, Space Science Centre, Sussex University, Falmer, Brighton BN1 9QH, UK.

M. F. Smith, Laboratory for Extraterrestrial Physics, NASA Goddard Space Flight Center, Greenbelt, MD 20771.

(Received July 21, 1994; revised October 20, 1994; accepted February 15, 1995) 